\title{
Numerically Absorbing Boundary Conditions for Quantum Evolution Equations
}

\author{
ANTON ARNOLD* \\ Fachbereich Mathematik, TU-Berlin, D-10623 Berlin, Germany and Department of Mathematics, Purdue University, \\ West Lafayette, IN 47907, USA
}

\begin{abstract}
Transparent boundary conditions for the transient Schrödinger equation on a domain $\Omega$ can be derived explicitly under the assumption that the given potential $V$ is constant outside of this domain. In $1 D$ these boundary conditions are non-local in time (of memory type). For the Crank-Nicolson finite difference scheme, discrete transparent boundary conditions are derived, and the resulting scheme is proved to be unconditionally stable. A numerical example illustrates the superiority of discrete transparent boundary conditions over existing ad-hoc discretizations of the differential transparent boundary conditions.

As an application of these boundary conditions to the modeling of quantum devices, a transient $1 D$ scattering model for mixed quantum states is presented.
\end{abstract}

Keywords: Schrödinger equation, transparent boundary conditions, absorbing boundary conditions, finite differences, discrete transparent boundary conditions, quantum device contacts.

\section{INTRODUCTION}

The formulation and implementation of physically reasonable and mathematically well-posed boundary conditions (BC) is one of the big open problems and challenges for transient simulations of semiconductor devices through quantum mechanical models. This paper is concerned with the construction and discretization of absorbing boundary conditions $(\mathrm{ABC})$ for the Schrödinger equation (SE)

$$
\begin{aligned}
i \hbar \psi_{t} & =-\frac{\hbar^{2}}{2} \Delta \psi+V(x, t) \psi, \quad x \in \mathbb{R}^{N}, t>0, \\
\psi(x, 0) & =\psi^{l}(x),
\end{aligned}
$$

where the electrostatic potential $V$ is assumed to be given. These BC's are derived from mathematical considerations, and they can be used as ingredients for modeling quantum contacts.

When numerically solving a whole-space evolution problem, the computation has to be confined to a finite domain by introducing artificial BC's. If the initial data is supported on this finite domain $\Omega$, one can approximate $\left.\psi\right|_{\Omega}$, the exact solution of the whole space problem restricted to $\Omega$, by solving the original problem only on $\Omega$, together with ABC's on $\partial \Omega$ If this approximate solution coincides on $\Omega$ with the exact solution, one refers to these BC's as transparent boundary conditions (TBC).

$A B C$ 's were first derived by Engquist and Majda for hyperbolic systems by requiring that outgoing waves can leave the computational domain without being reflected back in ([7]). Since then, these ideas have been adapted and refined for numerous applica-

\footnotetext{
*E-mail: arnold@math.tu-berlin.de
} 
tions. E.g., ABC's for the Wigner equation of quantum mechanics were obtained and analyzed in [16], [1], and they were used for transient simulations of resonant tunneling devices in [10].

The recent interest in finding reasonable BC's for the SE was mostly prompted by applications from outside of quantum mechanics. We remark that a Schrödinger-type equation naturally appears as a small-angle approximation to the Helmholtz equation in cylindrical coordinates. There, the radial variable $r$ plays the role of the time in (1.1), and the axial variable $z$ the role of the spatial variable $x$. In optical applications this model is referred to as the Fresnel equation ([17]), and in underwater acoustics it is called the parabolic equation ([15]).

In previous simulations, several heuristic BC's were introduced for the $\mathrm{SE}$, and they yield reasonable results for either short time calculations or a limited frequency range. These existing strategies include the introduction of an artificial absorption or attenuation layer close to the boundary, which can also be interpreted as adding a complex potential in (1.1) (see [11], [19], [20]). In another approach, the wave function $\psi$ is fitted to a plane wave close to the boundary ([8], [20]).

Our goal is to first derive (homogeneous) TBC's for the $2 D \mathrm{SE}$ under the two assumptions, that the initial data $\psi^{I}$ is supported in $\Omega$, and that the medium (here modeled by the potential $V$ ) is homogeneous and time-independent outside of $\Omega$. Otherwise information would be lost, and the whole space evolution could not be mimicked on the finite domain $\Omega$.

For the $1 D$ SE, the TBC was independently derived by several authors from various application fields ([14], [4], [9]).

As this general construction may be useful for many other applications, we will sketch this derivation in $\$ 2$ for the $1 D$ and $2 D$ Schrödinger equation, and analyze the well-posedness of the resulting initial boundary value problems (IBVP). In $\$ 3$ we derive and investigate an unconditionally stable numerical discretization of the $1 D$ problem, based on a Crank-Nicolson finite difference scheme. As an application of these TBC's we present in $\S 4$ a transient Schrödinger-Poisson scattering model for mixed quantum states.

\section{TRANSPARENT BOUNDARY CONDITIONS FOR THE SCHRÖDINGER EQUATION IN 1D AND 2D}

In this Section we will first derive the TBC's for the $2 D$ Schrödinger equation on the slab $\Omega=\{(x, y) \in$ $\left.\mathbb{R}^{2} 10<x<L\right\}$. We assume that the initial data $\psi^{I}$ is supported in $\Omega$, and that the given potential is constant outside: $V(x, y, t)=0$ for $x \leq 0, V(x, y, t)=V_{+}$for $x \geq L$.

We first cut the original whole space problem (1.1) into three subproblems, the interior problem on $\Omega$, and a left and right exterior problem. They are coupled by the assumption that $\psi, \psi_{x}$ are continuous across the boundary. The interior problem reads

$$
\begin{aligned}
i \hbar \psi_{t} & =-\frac{\hbar^{2}}{2} \Delta \psi+V(x, y, t) \psi, \\
& (x, y) \in \Omega, t>0, \\
\psi(x, y, 0) & =\psi^{I}(x, y), \\
\psi_{x}(0, y, t) & =\left(T_{-} \psi\right)(0, y, t), \\
\psi_{x}(L, y, t) & =\left(T_{+} \psi\right)(L, y, t) .
\end{aligned}
$$

Here, $T_{ \pm}$denote the Dirichlet-to-Neumann maps at the boundaries, and they are obtained by solving the two exterior problems:

$$
\begin{aligned}
i \hbar v_{t} & =-\frac{\hbar^{2}}{2} \Delta v, x<0, y \in \mathbb{R}, t>0, \\
v(x, y, 0) & =0, \\
v(0, y, t) & =\Phi(y, t), \quad y \in \mathbb{R}, t>0, \\
\left(T_{-} \Phi\right)(y, t) & :=v_{x}(0, y, t),
\end{aligned}
$$

and analogously for $T_{+}$. In (2.2) we also have to require that $v$ decays as $x \rightarrow-\infty$. Since the potential is constant in the exterior problems, we can solve them explicitly by the Fourier-Laplace method and thus obtain the two boundary operators $T_{ \pm}$needed in (2.1). The Fourier-Laplace transform of $v$ is

$$
\hat{v}(x, k, s)=\frac{1}{\sqrt{2 \pi}} \int_{0}^{\infty} \int_{\mathbb{R}} v(x, y, t) e^{-i k y} e^{-s t} d y d t,
$$

where we set $s=\eta+i \xi, \xi \in \mathbb{R}$, and $\eta>0$ is fixed, with the idea to later perform the limit $\eta \rightarrow 0$. Now the exterior problem (2.2) is transformed to

$$
\begin{aligned}
i \hbar s \hat{v} & =-\frac{\hbar^{2}}{2}\left(\hat{v}_{x x}-k^{2} \hat{v}\right), \quad x<0, k \in \mathbb{R}, \\
\hat{v}(0, k, s) & =\Phi(k, s) .
\end{aligned}
$$

Since its solutions have to decrease as $x \rightarrow-\infty$, we obtain 


$$
\hat{v}(x, k, s)=e^{\sqrt{k^{2}-2 i s / \hbar} x} \hat{\Phi}(k, s), \quad \operatorname{Re} \sqrt{k^{2}-2 i s / \hbar}>0 .
$$

Hence the transformed Dirichlet-to-Neumann operator $T_{-}$reads

$$
\begin{array}{r}
\widehat{T_{-} \Phi}(k, s)=\sqrt{\frac{2}{\hbar}} e^{-\frac{\pi}{4} i \sqrt{s+i \frac{\hbar}{2} k^{2}}} \hat{\Phi}(k, s), \\
\operatorname{Re} \sqrt{s+i \frac{\hbar}{2} k^{2}}>0,
\end{array}
$$

and $T_{+}$is calculated analogously.

Now we will first discuss the $1 D$ situation. Formally setting $k=0$ in (2.6), the left TBC is obtained by an inverse Laplace transform:

$$
\psi_{x}(0, t)=\sqrt{\frac{2}{\hbar \pi}} e^{-\frac{\pi}{4} i} \frac{d}{d t} \int_{0}^{t} \frac{\psi(0, \tau)}{\sqrt{t-\tau}} d \tau
$$

Similarly, the right TBC is derived as

$$
\begin{aligned}
& \psi_{x}(L, t)= \\
& \quad-\sqrt{\frac{2}{\hbar \pi}} e^{-\frac{\pi}{4} i} e^{-i V_{+} t / \hbar} \frac{d}{d t} \int_{0}^{t} \frac{\psi(L, \tau) e^{i V_{+} \tau / \hbar}}{\sqrt{t-\tau}} d \tau .
\end{aligned}
$$

These BC's are non-local in $t$ and of memory-type, thus requiring the storage of all the past history at the boundary in a numerical discretization.

We remark that the term

$$
\frac{1}{\sqrt{\pi}} \frac{d}{d t} \int_{0}^{t} \frac{\psi(\tau)}{\sqrt{t-\tau}} d \tau=: \sqrt{\frac{d}{d t}} \psi
$$

can be interpreted as a fractional $\frac{1}{2}$ time derivative. A simple calculation also shows that (2.7) is equivalent to the impedance boundary condition derived in [14] :

$$
\psi(0, t)=\sqrt{\frac{\hbar}{2 \pi}} e^{\frac{\pi}{4} i} \int_{0}^{t} \frac{\psi_{x}(0, \tau)}{\sqrt{t-\tau}} d \tau .
$$

From the used construction, it is clear that the $1 D$ Schrödinger equation with the TBC's (2.7) and (2.8) has a solution. For regular enough initial data, e.g. $\psi^{I}$ $\in H_{0}^{1}(0, L)$, the whole space solution $\psi(x, t)$ will satisfy the TBC's at least in a weak sense. The uniqueness of the solution is, however, not trivial. In order to prove uniform boundedness of $\|\psi(., t)\|_{L^{2}(0, L)}$ in $t$ we will need the following simple lemma.
Lemma 2.1 For any $T>0$, let $u \in H^{1 / 4}(0, T)$ with the extension $u(t)=0$ for $t>T$. Then

$$
\operatorname{Re}\left\{e^{\frac{i \pi}{4}} \int_{0}^{\infty} \bar{u}(t) \frac{d}{d t}\left[\int_{0}^{t} \frac{u(\tau)}{\sqrt{t-\tau}} d \tau\right] d t\right\} \geq 0 .
$$

Proof : Using the Plancherel equality for the Laplace transform, the 1.h.s. of (2.11) equals

$$
\frac{1}{2 \pi} \operatorname{Re}\left\{e^{\frac{i \pi}{4}} \int_{\mathbb{R}} \overline{\hat{u}}(i \xi) \sqrt[+]{i \xi} \hat{u}(i \xi) d \xi\right\} \geq 0 .
$$

Here, $\sqrt[+]{ }$ the branch of the square root with non-negative real part.

A straight forward calculation with the $1 D$ Schrödinger equation now shows

$$
\|\psi(t)\|_{L^{2}(0, L)} \leq\left\|\psi^{\prime}\right\|_{L^{2}(0, L)}, \quad t>0
$$

and this implies uniqueness of the solution to the Schrödinger IBVP. In this calculation one needs the estimate (2.11) for the left boundary term, and an analogous estimate for the right boundary. (2.13) reflects the fact that some of the initial mass or particle density $n(x, t)=|\psi(x, t)|^{2}$ leaves the computational domain $[0, L]$ during the evolution. In the whole space problem $\|\psi(t)\|_{L^{2}(\mathbb{R})}$ is of course conserved.

We now return to the $2 D$ problem on the vertical strip $0<x<L$. For this case we derived the TBC in (2.6). After inverse Fourier and Laplace transformation, this TBC is a convolution in $t$ and $y$, generalizing the explicit $1 D$ representation (2.7). From a numerical point of view, an implementation of this non-local (in $t$ and $y$ ) BC would be rather costly. It is therefore desirable to first approximate this TBC by a BC that is at least local in $y$.

In order to motivate our approximation, we first sketch an alternative, heuristic derivation of the $2 D$ TBC (2.6) at $x=0$. We consider left traveling plane wave solutions to the free SE (since the potential $V=0$ close to the left boundary):

$$
\psi(x, y, t)=\exp \left[i\left(-\sqrt{\frac{2 \omega}{\hbar}-k^{2}} x+k y-\omega t\right)\right],
$$

with $\omega>0$ and $k$ fixed. At $x=0$ this plane wave satisfies

$$
\psi_{x}=-i \sqrt{\frac{2 \omega}{\hbar}-k^{2}} \psi
$$


and this coincides with (2.6) if we set $\omega=i$ s. One easily sees that $\theta$, the impact angle of the plane wave at the boundary (measured towards the normal) is given by $\sin ^{2} \theta=\hbar k^{2} /(2 \omega)$. If outgoing waves hit the boundary almost orthogonally $(\theta \approx 0)$, it is a standard strategy (see [7]) to use a Taylor approximation in $\theta$ of the symbol of the pseudo-differential operator $T_{-}$(see (2.6)):

$$
\sqrt{s+i \frac{\hbar}{2} k^{2}}=\sqrt{s} \cos \theta \approx \sqrt{s} \text { or } \approx \sqrt{s}\left(1+\frac{i \hbar}{4} \frac{k^{2}}{s}\right) .
$$

In lowest order this yields a first order $\mathrm{ABC}$ for the $2 D$ SE at $x=0$ :

$$
\psi_{x}(0, y, t)=\sqrt{\frac{2}{\hbar \pi}} e^{-\frac{\pi}{4} i} \frac{\partial}{\partial t} \int_{0}^{t} \frac{\psi(0, y, \tau)}{\sqrt{t-\tau}} d \tau
$$

This BC essentially neglects $2 D$ effects at the boundary, and the well-posedness of the resulting IBVP carries over from the $1 D$ situation. On finite time intervals, this solution can be expected to be a reasonable approximation to the whole space solution, under the assumption $\theta \approx 0$.

In the next order approximation from (2.16) we first get after multiplying by $\sqrt[+]{s}$ :

$$
\sqrt[+]{s} \hat{\psi}_{x}(0, k, s)=\sqrt{\frac{2}{\hbar}} e^{-\frac{\pi}{4} i}\left(s+\frac{i \hbar k^{2}}{4}\right) \hat{\psi}(0, k, s) .
$$

And by inverse Laplace-transformation, the second order $\mathrm{ABC}$ then reads

$$
\sqrt{\frac{\partial}{\partial t}} \psi_{x}=\sqrt{\frac{2}{\hbar}} e^{-\frac{\pi}{4} i}\left(\psi_{t}-\frac{i \hbar}{4} \psi_{y y}\right), \quad x=0 .
$$

We conjecture that the resulting IBVP is well-posed; this problem will be analyzed in a forthcoming paper.

\section{DISCRETIZATION OF THE TRANSPARENT BOUNDARY CONDITION IN 1D}

In this Section we will discuss a discretization of the $1 D$ TBC (2.7) based on the Crank-Nicolson finite difference scheme for the SE. With the uniform grid points $x_{j}=j \Delta x, t_{n}=n \Delta t$, and the approximations $\psi_{j}^{n} \sim \psi\left(x_{j}, t_{n}\right)$ this scheme reads for the whole space problem

$$
\begin{array}{r}
r\left(\psi_{j}^{n+1}-\psi_{j}^{n}\right)=\psi_{j+1}^{n+1}-2 \psi_{j}^{n+1}+\psi_{j-1}^{n+1}+\psi_{j+1}^{n} \\
-2 \psi_{j}^{n}+\psi_{j-1}^{n}+w V_{j}^{n+\frac{1}{2}}\left(\psi_{j}^{n+1}+\psi_{j}^{n}\right),
\end{array}
$$

with

$$
r=-\frac{4 i \Delta x^{2}}{\hbar \Delta t}, w=-\frac{2 \Delta x^{2}}{\hbar^{2}}, V_{j}^{n+\frac{1}{2}}=V\left(x_{j}, t_{n+\frac{1}{2}}\right) .
$$

For our analysis, one of the main advantages of this second order (in $\Delta x$ and $\Delta t$ ) scheme is, that it is unconditionally stable, and it preserves the discrete $L^{2}$-norm : $\left\|\psi^{n}\right\|_{2}^{2}=\Delta x \sum_{j \in \mathbb{Z}}\left|\psi_{j}^{n}\right|^{2}$

We remark that most existing discretization schemes for the $1 D$ Schrödinger equation with TBC's are also based on Crank-Nicolson finite differences ([4], [13], [15]). In [17] a semi-discrete TBC for the $1 D$ SE with time-dependent potentials in the exterior domains (i.e., $V_{+}=V_{+}(t)$ ) was derived. For constant potentials, the obtained method (also Crank-Nicolson) is related to our ideas.

The delicate question is how to discretize the convolution (2.7) with its singular kernel. In [13], Mayfield used the approximation

$$
\int_{0}^{t_{N}} \frac{\psi_{x}\left(0, t_{N}-\tau\right)}{\sqrt{\tau}} d \tau \approx \frac{1}{\Delta x} \sum_{n=0}^{N-1}\left(\psi_{1}^{N-n}-\psi_{0}^{N-n}\right) \int_{t_{n}}^{t_{n+1}} \frac{d \tau}{\sqrt{\tau}}
$$

for the TBC in the equivalent formulation (2.10). For the resulting scheme she obtained the following result:

Theorem 3.1 ([13]) : The discretization scheme (3.1), (3.2) is stable, if and only if

$$
4 \pi \hbar \frac{\Delta t}{\Delta x^{2}} \in \bigcup_{j \in \mathbb{N}_{0}}\left[(2 j+1)^{-2},(2 j)^{-2}\right] .
$$

This shows that the chosen boundary discretization destroys the unconditional stability of the underlying Crank-Nicolson scheme.

Our aim is to derive a different boundary discretization of (2.7) which gives an unconditionally stable scheme and a discrete analogue of (2.13), the uniform boundedness of the continuous $L^{2}$-norm. When considering the discretization of TBC's, it should be a 
standard strategy to derive the discrete TBC's of the fully discretized problem, rather then attempting to discretize the differential TBC. Discrete TBC's not only completely avoid any numerical reflections at the boundary, but their numerical stability is often also better-behaved, as it is the case here. A comparison of these two strategies for a $1 D$ wave propagation problem is given in [6].

To derive the discrete TBC we will now mimic the derivation from $\$ 2$ on a discrete level, and we will again only consider the left BC. The idea is to explicitly solve the discrete exterior problems. The $Z$-transform of the sequence $\left\{\psi_{j}^{n}\right\}, n \in \mathbb{N}_{0}$, with $j$ considered fixed, is defined as the Laurent series

$$
Z\left\{\psi_{j}^{n}\right\}=\hat{\psi}_{j}(z):=\sum_{n=0}^{\infty} \psi_{j}^{n} z^{-n}
$$

In analogy to the continuous problem we assume that the potential and initial data vanish for $x \leq 0$ :

$$
V_{j}^{n+\frac{1}{2}}=0 \text { for } j \leq 0, \quad \psi_{j}^{0}=0 \text { for } j \leq 1 .
$$

Then, the $Z$-transformed Crank-Nicolson scheme for the left exterior problem reads

$\hat{\psi}_{j+1}-\left(2+r \frac{z-1}{z+1}\right) \hat{\psi}_{j}+\psi \hat{j}_{j-1}=0, \quad j \leq 0$.

This difference equation has the two linearly independent solutions

$$
\begin{gathered}
\hat{\psi}_{j}=\alpha^{j}, \quad j \leq 1 ; \\
\alpha_{1,2}(z)=1+\frac{r}{2} \frac{z-1}{z+1} \pm \frac{r}{2} \frac{1}{z+1} \sqrt{z^{2}\left(1+\frac{4}{r}\right)-2 z+1-\frac{4}{r}} .
\end{gathered}
$$

Since we need decreasing modes as $j \rightarrow-\infty$, we have to require $\left|\alpha_{1}(z)\right|>1$.

The (Z-transformed) discrete TBC is now obtained as $\hat{\psi}_{1}(z)=\alpha_{1}(z) \hat{\psi}_{0}(z)$ which remains to be inverse transformed. In a tedious calculation this can be achieved explicitly:

Theorem 3.2 The left $(a t j=0)$ and right $(a t j=J)$ discrete TBC's for the Crank-Nicolson discretization (3.1) of the $1 D$ Schrödinger equation are

$$
\begin{aligned}
& \psi_{1}^{n}=\sum_{k=1}^{n} \psi_{0}^{k} l_{0}^{n-k}- \psi_{1}^{n-1} \\
& \psi_{J-1}^{n}=\sum_{k=1}^{n} \psi_{J}^{k} l_{J}^{n-k}-\psi_{J-1}^{n-1}, \quad n \geq 1,
\end{aligned}
$$

with

$$
\begin{gathered}
l_{j}^{n}=\left(1-i \frac{\rho}{2}+\frac{\sigma_{j}}{2}\right) \delta_{n}^{0}+\left(1+i \frac{\rho}{2}+\frac{\sigma_{j}}{2}\right) \delta_{n}^{1}+ \\
\alpha_{j} e^{-i n \varphi_{j}} \frac{P_{n}\left(\mu_{j}\right)-P_{n-2}\left(\mu_{j}\right)}{2 n-1}, \quad j=0, J, \quad \\
\rho=\frac{4 \Delta x^{2}}{\hbar \Delta t}, \quad \varphi_{j}=\arctan \frac{2 \rho\left(\sigma_{j}+2\right)}{\rho^{2}-4 \sigma_{j}-\sigma_{j}^{2}}, \quad \sigma_{j}=\frac{2 \Delta x^{2}}{\hbar^{2}} V_{j} \\
\mu_{j}=\frac{\rho^{2}+4 \sigma_{j}+\sigma_{j}^{2}}{\sqrt{\left(\rho^{2}+\sigma_{j}^{2}\right)\left(\rho^{2}+\left[\sigma_{j}+4\right]^{2}\right)}}, \\
\alpha_{j}=\frac{i}{2} \sqrt[4]{\left(\rho^{2}+\sigma_{j}^{2}\right)\left(\rho^{2}+\left[\sigma_{j}+4\right]^{2}\right)} e^{i \varphi_{j} / 2}
\end{gathered}
$$

where $P_{n}$ denotes the Legendre polynomials $\left(P_{-1} \equiv P_{-2} \equiv\right.$ $0)$, and $\delta_{n}^{j}$ the Kronecker symbol.

The $P_{n}$ only have to be evaluated at the two values $\mu_{0}$ and $\mu_{J}$, and hence the numerically stable recursion formula for the Legendre polynomials can be used. $l_{0}^{n}, l_{j}^{n}=O\left(n^{-3 / 2}\right)$ which agrees with the decay of the convolution kernel in the differential TBC's (2.7), (2.8) (after an integration by parts).

The system matrix of the implicit scheme (3.1), (3.8) is diagonal-dominant and hence invertible. To prove stability of the scheme, one can derive a discrete analogue of Lemma 2.1 and (2.13). We then have the main result of this Section:

Theorem 3.3 The solution of the discretized SE (3.1) with the discrete TBC's (3.8) is uniformly bounded

$$
\left\|\psi^{n}\right\|_{2}^{2}:=\Delta x \sum_{j=1}^{J-1}\left|\psi_{j}^{n}\right|^{2} \leq\left\|\psi^{0}\right\|_{2}^{2}, \quad n \geq 1,
$$

and the scheme is thus unconditionally stable.

It can also be shown that (3.8) is a consistent discretization of the differential BC's (2.7), (2.8). The details of the numerical analysis will be presented in [2]. Fig. 1 shows a simulation of a right traveling Gaussian beam evolving under the free Schrödinger equation ( $\hbar=1)$ with the rather course discretization $\Delta x=0.00625, \Delta t=0.00002$. Discretizing the analytic 
TBC's via (3.2) introduces strong numerical reflections, while our discrete TBC's (3.8) recovers the smooth solution.
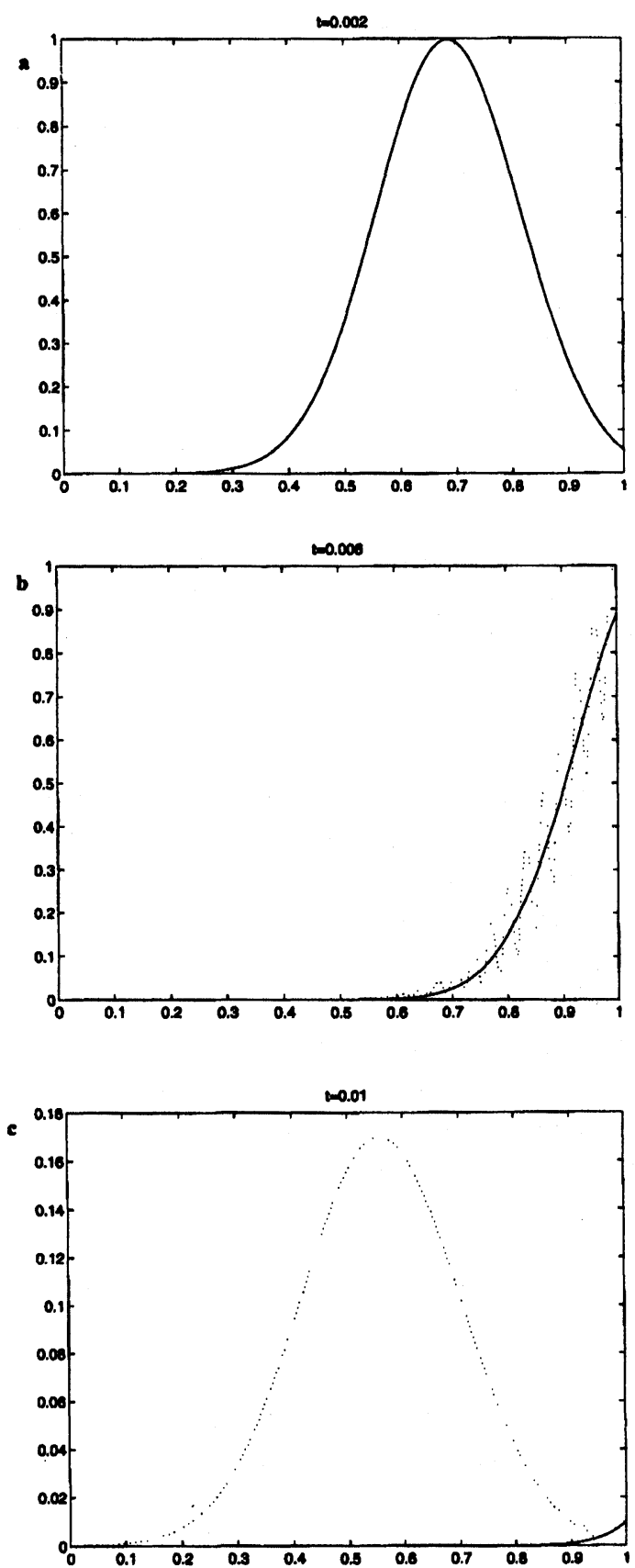

FIGURE $1 \nmid \psi(x, t) \mid$ for a right traveling Gaussian beam at three consecutive times with discrete TBC's (3.8) (solid) and the discretized analytic TBC's (3.2) (dotted)

\section{A TRANSIENT SCHRÖDINGER-POISSON SCATTERING MODEL WITH TBC'S}

TBC's for the $2 D$ steady state SE have been used in [12] to model current carrying states in quantum devices. In $1 D$, these $\mathrm{BC}$ 's are the steady state analogue of (2.7), (2.8). A similar $1 D$ Schrödinger-Poisson scattering model has been analyzed mathematically in [5].

The (transient) $1 D$ TBC's of $\$ 2$ can of course only be used for simulating the time evolution of a pure state wave function $\psi$. For the application to transient semiconductor device modeling, we will now present a self-consistent Schrödinger-Poisson model for mixed quantum states with inhomogeneous TBC's.

The (homogeneous) TBC (2.7) was derived for modeling the situation where an initial wave function is supported in the computational domain $[0, L]$, and it is leaving this domain without being reflected back. If an incoming wave function $\psi_{i n}(t)$ is given at the left boundary (e.g. a right traveling plane wave), the inhomogeneous TBC

$$
\left[\psi(0, t)-\psi_{\text {in }}(t)\right]_{x}=\sqrt{\frac{2}{\hbar}} e^{-\frac{\pi}{4} i} \sqrt{\frac{\partial}{\partial t}}\left[\psi(0, t)-\psi_{\text {in }}(t)\right]
$$

has to be prescribed at $x=0$.

In our $1 D$ transient scattering model the state of the electrons is represented by the sum of two density matrices $\rho_{1,2}(x, y, t) . \rho_{1}$ describes the contribution of the initial state $\rho^{I}$, which is supported in the considered domain $[0, L]^{2}$, and $\rho_{2}$ is the density matrix for the incoming wave packets from the boundary.

Since we assume $\rho_{1} \in L^{2}\left((0, L)^{2}\right)$, it can be factorized into pure state wave functions as

$$
\rho_{1}(x, y, t)=\sum_{j \in \mathbb{N}} \lambda_{j} \psi_{j}(x, t) \bar{\psi}_{j}(y, t),
$$

and they satisfy

$$
\begin{aligned}
i \hbar \partial_{t} \psi_{j}= & -\frac{\hbar^{2}}{2} \partial_{x x} \psi_{j}+V(x, t) \psi_{j}, \\
& 0<x<L, t>0 \\
\psi_{j}(x, 0)= & \psi_{j}^{J}(x), \quad \operatorname{supp}\left(\psi_{j}^{I}\right) \subset(0, L), \\
V(x, t)= & 0 \quad \text { for } x \leq 0, \quad \text { for } x \geq L, \\
& V(x, t)=V_{+} \quad \text { or }
\end{aligned}
$$


together with the homogeneous TBC's (2.7), (2.8) for each $\psi_{j}, j \in \mathbb{N}$.

For simplicity we assume that there is a constant incoming wave packet, only at the left boundary, that consists of the right-traveling plane waves $\exp \left[i / \hbar\left(k x-k^{2} t / 2\right)\right], k>0$. Each wave vector $k$ of this incoming packet is associated with a weight $g(k) \geq 0$. The density matrix $\rho_{2}$ can then be represented as

$$
\rho_{2}(x, y, t)=\int_{0}^{\infty} g(k) \psi_{k}(x, t) \bar{\psi}_{k}(y, t) d k
$$

and these wave functions $\psi_{k}, k>0$ satisfy

$$
\begin{aligned}
i \hbar \partial_{t} \psi_{k} & =-\frac{\hbar^{2}}{2} \partial_{x x} \psi_{k}+V(x, t) \psi_{k}, \\
\psi_{k}(x, 0) & =0 .
\end{aligned}
$$

At the left boundary $x=0$ they satisfy the inhomogeneous TBC (4.1) with

$$
\psi_{k, i n}(t)=\exp \left[-\frac{i}{\hbar} \frac{k^{2}}{2} t\right]
$$

and at the right boundary the homogeneous TBC (2.8).

The total particle density associated with the state $\rho_{1}+\rho_{2}$ is

$$
\begin{aligned}
n(x, t)= & \sum_{j \in \mathbb{N}} \lambda_{j}\left|\psi_{j}(x, t)\right|^{2} \\
& +\int_{0}^{\infty} g(k)\left|\psi_{k}(x, t)\right|^{2} d k, \quad 0<x<L .
\end{aligned}
$$

In a self-consistent model, the potential $V(x, t)$ would then be obtained from the Poisson equation:

$$
\begin{aligned}
V_{x x} & =-n, \quad 0<x<L, \\
V(0, t) & =0, \quad V(L, t)=V_{+} .
\end{aligned}
$$

We remark that the scattering model of [5] is the steady state analogue of the above model (4.2)-(4.8).

The presented model was obtained in collaboration with N. Ben Abdallah, and a mathematical analysis of this model will be given in [3].

\section{Acknowledgements}

The author thanks N. Ben Abdallah, J. Douglas, and M. Peszynska for stimulating discussions on this topic. This research was partially supported by the grants ERBCHRXCT930413 from the EC, MA 1662/2-1 from the DFG, and the NSF under Grant No. DMS-9500852.

\section{References}

[1] A. Arnold, Math. Meth. Num. Anal. 7 (1994) 853-872.

[2] A. Arnold, M. Peszyńska, Absorbing boundary conditions for the Schrödinger equation : an unconditionally stable discretization, in preparation (1995).

[3] A. Arnold, N. Ben Abdallah, On a transient Schrödinger-Poisson model with absorbing boundaries, in preparation (1995).

[4] V.A. Baskakov, A.V. Popov, Wave Motion 14 (1991) 123-128.

[5] N. Ben Abdallah, P. Degond, P.A. Markowich, On a one-dimensional Schrödinger-Poisson scattering model, submitted (1995).

[6] J. Douglas Jr., J.E. Santos, D. Sheen, L.S. Bennethum, Math. Mod. and Meth. in Appl. Sc. (1993) 171-194.

[7] B. Engquist, A. Majda, Math. Comp. 31 (1977) 629-651.

[8] G.R. Hadley, IEEE J. Quantum Electron. 28 (1992) 363-370.

[9] J.R. Hellums, W.R. Frensley, Phys. Rev. B 49 (1994) 2904-2906.

[10] N. Kluksdahl, A.M. Kriman, D.K. Ferry, C. Ringhofer, Selfconsistent study of the resonant tunneling diode, Phys. Rev. B 39 (1989) 7720-7735.

[11] R. Kosloff, D. Kosloff, J. Comput. Phys. 63 (1986) 363-376.

[12] C.S. Lent, D.J. Kirkner, J. Appl. Phys. 67 (1990) 6353-6359.

[13] B. Mayfield, Non-local boundary conditions for the Schrödinger equation, Ph.D. thesis, University of Rhode Island, Providence, RI (1989).

[14] J.S. Papadakis, Impedance formulation of the bottom boundary condition for the parabolic equation model in underwater acoustics, NORDA Parabolic Equation Workshop, NORDA Tech. Note 143 (1982).

[15] J.S. Papadakis, M.I. Taroudakis, P.J. Papadakis, B. Mayfield, J. Acoust. Soc. Am. 92 (1992) 2030-2038.

[16] C. Ringhofer, D. Ferry, N. Kluksdahl, Transp. Theory and Stat. Phys. 18 (1989) 331-346.

[17] F. Schmidt, P. Deuflhard, Computers Math. Applic. 29 (1995) 53-76.

[18] D.J. Thomson, M.E. Mayfield, J. Comput. Acoust. 2 (1994) 113-132.

[19] D. Yevick, B. Hermansson, IEEE J. Quantum Electron. 25 (1989) 221-229.

[20] D. Yevick, J. Yu, Y. Yayon, J. Opt. Soc. Am. A 12 (1995) 107-110.

\section{Biography}

Anton Arnold is C2-Associate Professor of Mathematics at the Technical University of Berlin. His research interests include quantum transport equations, semiconductor device modeling, and hyperbolic PDE's. 

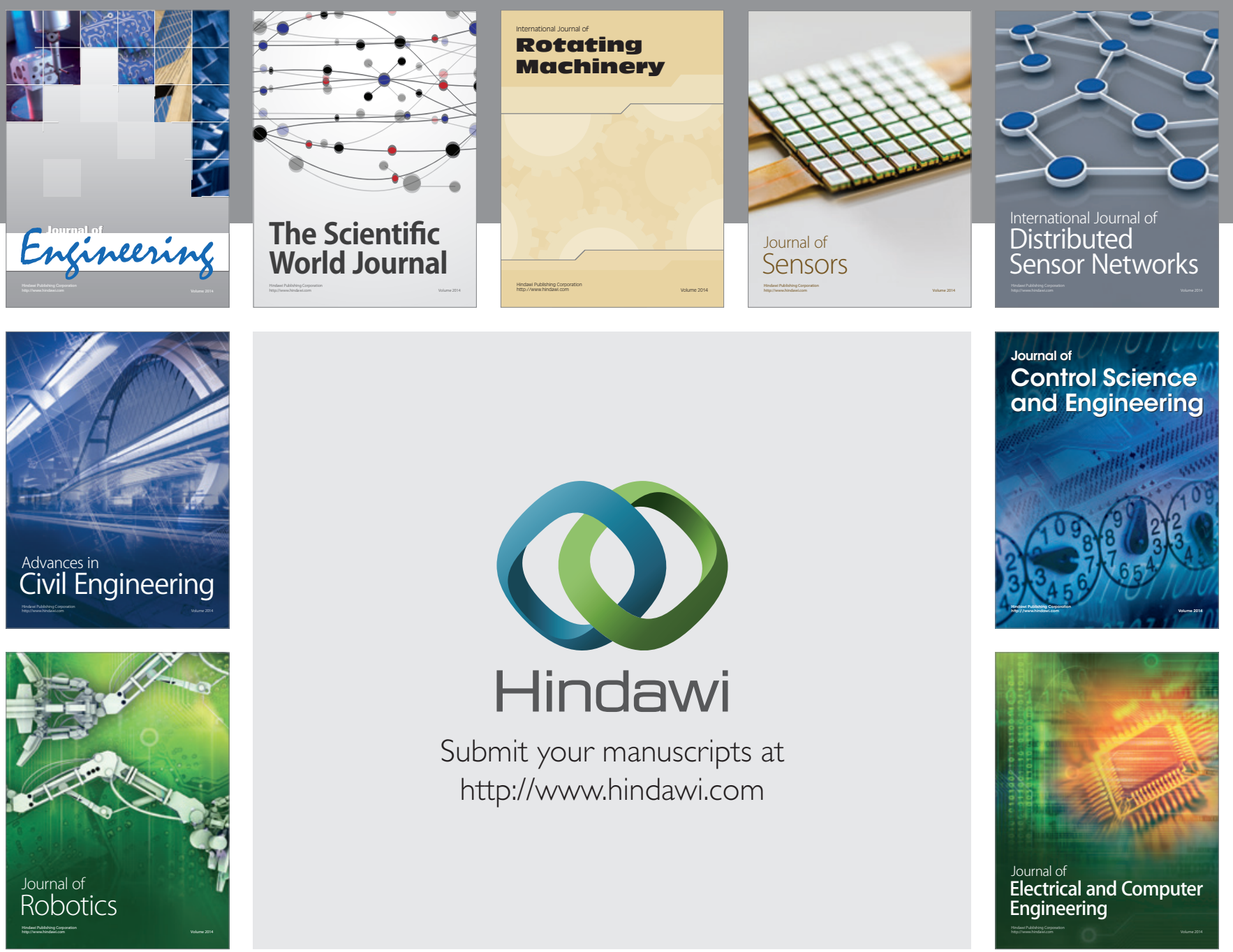

Submit your manuscripts at

http://www.hindawi.com
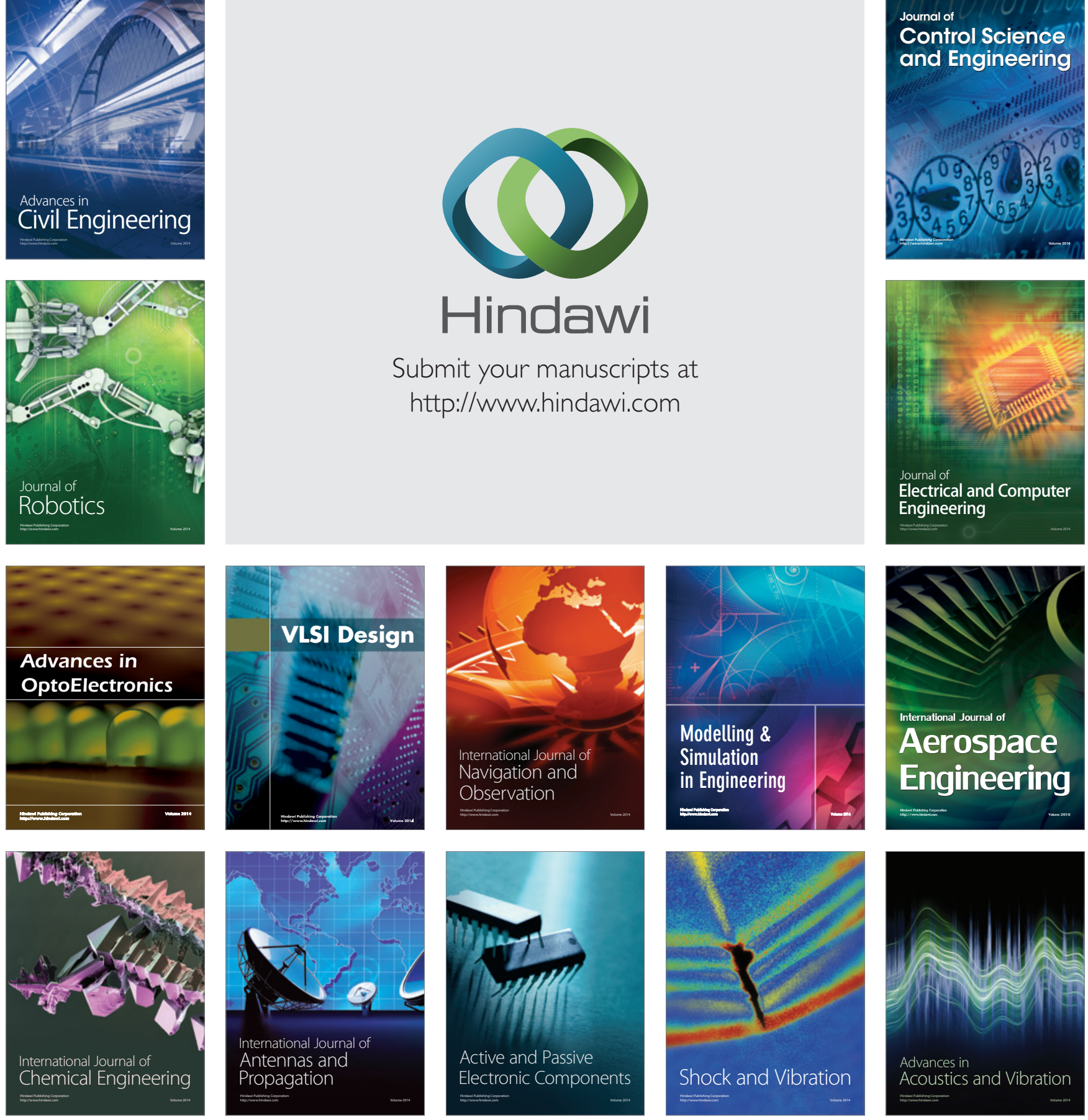